\title{
Genersatztherapie bei genetisch bedingter Zapfenblindheit
}

\author{
Gene Replacement Therapy in Achromatopsia Type 2
}

Autoren

Institut
R. Mühlfriedel, N. Tanimoto, M. W. Seeliger

Forschungsinstitut für Augenheilkunde, Department für Augenheilkunde, Eberhard Karls-Universität, Tübingen

\author{
Schlüsselwörter \\ - rAAV-basierte Genersatz- \\ therapie \\ - nicht invasive Diagnostik- \\ methoden \\ - Achromatopsie \\ - CNGA3-Kanalprotein \\ - erbliche Netzhaut- \\ erkrankungen

\section{Key words \\ non-invasive diagnostic methods \\ - achromatopsia \\ - CNGA3 channel protein \\ - hereditary retinal diseases} \\ - AAV-mediated gene therapy
}

eingereicht 3.2.2014

akzeptiert 11.2.2014

\section{Bibliografie}

DOI http://dx.doi.org/ 10.1055/s-0034-1368180

Klin Monatsbl Augenheilkd

2014; 231: 232-240 @ Georg

Thieme Verlag KG Stuttgart .

New York · ISSN 0023-2165

Korrespondenzadresse Dr. Regine Mühlfriedel

Forschungsinstitut für Augenheilkunde, Department für Augenheilkunde

Eberhard Karls-Universität 72076 Tübingen

Tel.: ++49/(0)7071/2987784

regine.muehlfriedel@

uni-tuebingen.de

\section{License terms}

\section{(ब) (1) $\Theta \circledast$}

\section{Zusammenfassung \\ $\nabla$}

Achromatopsie ist eine autosomal-rezessiv vererbte Netzhauterkrankung, die durch den angeborenen kompletten Funktionsverlust der Zapfenphotorezeptoren charakterisiert ist. Etwa 80\% der Patienten weisen Mutationen in der $\alpha$ - oder $\beta$-Untereinheit (A3 oder B3) des cGMP-gesteuerten Kationenkanals CNG (cyclic nucleotide-gated channel) der Zapfenphotorezeptoren auf. Homolog zum humanen Krankheitsbild zeigen CNGA3defiziente Mäuse einen zapfenspezifischen Funktionsverlust, der zur Degeneration der betroffenen Zapfenphotorezeptorzelle führt. Am Forschungsinstitut für Augenheilkunde in Tübingen gelang es nun erstmalig im Tiermodell für Achromatopsie ACHM2, eine Netzhauterkrankung umfassend zu therapieren. Im vorliegenden Artikel werden der auf subretinaler Injektion von rekombinanten Adeno-assoziierten Viren (rAAV) basierende Ansatz im Detail dargelegt und die eingesetzten nicht-invasiven Diagnostikmethoden ERG, SLO und OCT zur Erfolgs- und Qualitätskontrolle beschrieben. Der nachweisbare Therapieerfolg zeigt sich in einem Funktionsgewinn des Zapfensystems und in einer weiterführenden neuronalen Verarbeitung des retinalen Signals bis hin zu einem zapfendominierten Verhalten der behandelten Tiere. Die herausragenden Ergebnisse sind Ausgangspunkt für die erste humane Translation einer Gentherapie für Achromatopsie in Deutschland.

\section{Einleitung}

\section{$\nabla$}

Die menschliche Netzhaut mit ca. 125 Mio. hoch spezialisierten lichtempfindlichen Sinneszellen, den Photorezeptoren, ist der Ort, wo der Sehvorgang seinen Anfang nimmt. Die Leistungsfähigkeit des visuellen Systems wird durch die Arbeitsteilung zweier Arten von Photorezeptoren bestimmt: die Stäbchen, die ca. 95\% aller Photo-

\section{Abstract \\ $\nabla$}

Achromatopsia is an autosomal recessive inherited retinal disease caused by a complete loss of cone photoreceptor function. About $80 \%$ of achromatopsia patients show mutations in the alpha or beta subunit (A3 and B3) of the CGMP controlled cation channel CNG (cyclic nucleotide-gated channel) of cone photoreceptors. Homologous to the human disease, CNGA3 deficient mice reveal a loss of cone specific functionality leading to degeneration of affected cone photoreceptors. The Institute for Ophthalmic Research in Tübingen has now succeeded in curing achromatopsia ACHM2 in an animal model. In this article, we explain the recombinant adeno-associated virusbased approach in detail. Furthermore, applied non-invasive diagnostic techniques for quality and success control, ERG, SLO and OCT, are described. The success of the therapy is indicated by a restored cone photoreceptor function as well as the neuronal processing of retinal signals resulting in a specific, cone-mediated behaviour. The outstanding results derived from the animal model are the starting point for the first human translation of a gene therapy for achromatopsia in Germany. 


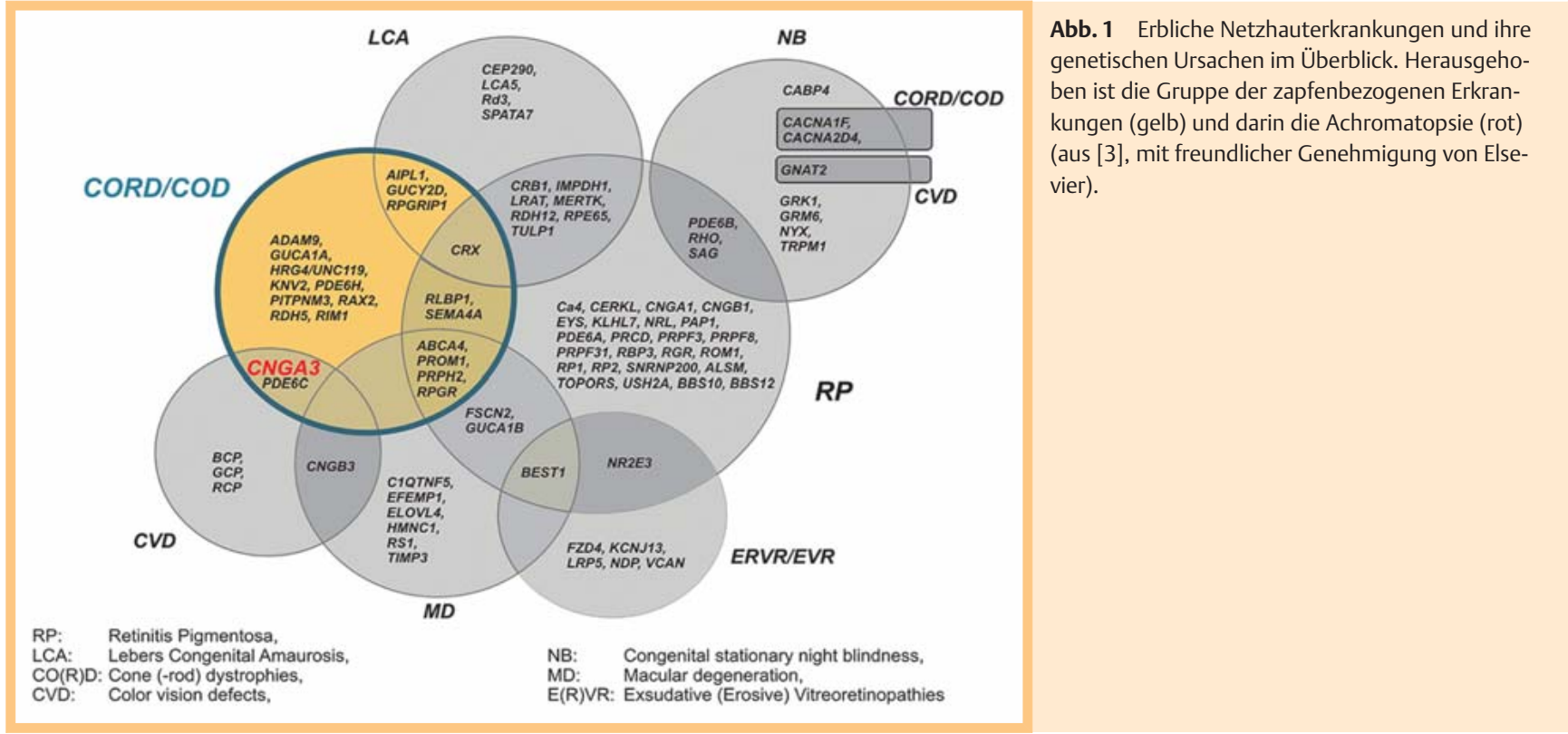

Im Bereich des Netzhautzentrums, der Fovea, bestimmen die Zapfen durch ihre hohe Packungsdichte und ihre besondere Verschaltung auf die weiterführenden Neurone das räumliche und zeitliche Auflösungsvermögen und sind daher unter Normalbedingungen für die erreichte Sehschärfe verantwortlich. Drei verschiedene Zapfentypen (Blau-, Grün- und Rotzapfen) mit unterschiedlicher spektraler Empfindlichkeit erschließen einen Spektralbereich des Lichtes von ca. 400-760 nm und vermitteln damit eine zusätzliche visuelle Qualität, die Farbwahrnehmung und Farbdifferenzierung. Aufgrund der herausragenden Bedeutung der Photorezeptoren gehen viele Einschränkungen des visuellen Systems auf Erkrankungen zurück, bei denen die Funktion der Photorezeptoren beeinträchtigt ist. Bei erblichen Netzhauterkrankungen kommt es oft zu genetisch bedingten Ausfällen photorezeptorspezifischer Gene, wodurch eine große Zahl von klinisch und ursächlich abgrenzbaren Krankheitsbildern entsteht, die jedes für sich zu den seltenen Erkrankungen gehört. In ihrer Gesamtheit und mit einer Prävalenz von 1:2500 sind die erblichen Netzhauterkrankungen jedoch ein klinisch bedeutsamer Erkrankungstypus - insbesondere auch im Hinblick auf die damit einhergehenden Einschränkungen in der Erwerbsfähigkeit und dem Verlust an Lebensqualität für die Betroffenen $[1,2]$. Charakteristisch für erblich bedingte Netzhauterkrankungen sind Funktionsausfälle in der Reizaufnahme und Reizweiterleitung innerhalb der Netzhaut, die zur Reduktion des Sehvermögens bis zur Blindheit führen. Bis heute wurden Mutationen in fast 180 Genen identifiziert, die für die verschiedenen Formen erblicher Netzhautdystrophien verantwortlich sind ([3], https:// sph.uth.edu/Retnet). Bisher gibt es noch keine etablierte Therapie für Netzhauterkrankungen beim Menschen. Die Fortschritte, die gerade im Bereich der Gentherapie innerhalb der letzten Jahre erzielt werden konnten, bieten nun neue Perspektiven. Es wurden sichere therapeutische Vektoren generiert, basierend auf rekombinanten Adeno-assoziierten Viren (rAAV), die für den Gentransfer in die Netzhaut verwendet werden können [4]. Weltweit wurde mithilfe dieser rAAV-basierten Vektorsysteme u.a. Gentherapieansätze für Achromatopsie am Tiermodell etabliert, die im vorliegenden Übersichtsartikel gegenübergestellt und diskutiert werden. Am Beispiel einer Genersatztherapie für Achro- matopsie (ACHM2), bedingt durch einen Zapfenkanaldefekt, werden die Möglichkeiten, Herausforderungen und anstehenden Aufgaben einer humanen Translation erläutert.

\section{Klinische Manifestation der Achromatopsie $\nabla$}

Die komplette Form der Achromatopsie ist eine autosomal-rezessiv vererbliche Netzhautdysfunktion, bei der die Funktion des Stäbchensystems nicht beeinträchtigt ist, jedoch die Zapfenphotorezeptoren völlig funktionslos sind ( $\bullet$ Abb. 1). Erkrankte leiden unter starken Einschränkungen ihrer Sehkraft, wie z.B. einer Reduktion der Sehschärfe auf $<10 \%$ des Normalen, einer starken Blendungsempfindlichkeit und einem völligen Verlust der Farbwahrnehmung. Des Weiteren entwickelt sich aufgrund der schlechten Sehschärfe ein Nystagmus (i.d. R. dauernde horizontale Pendelbewegung der Augen), der das Sehen zusätzlich erschwert [5]. Die Erkrankung ist mit einer Prävalenz von 1:30000 beschrieben worden [6-8]. Die Symptome der Achromatopsie, hauptsächlich die Blendungsempfindlichkeit, werden versucht durch Hilfsmittel wie ständiges Tragen von dunkel gefärbten Spezialkontaktlinsen $[9,10]$ oder Sonnenbrillen [11] zu lindern.

\section{Genetik der Achromatopsie \\ $\nabla$}

Nach heutigem Wissensstand sind 5 Gene bekannt, die ursächlich das Krankheitsbild der Achromatopsie im Patienten bedingen. Diese Gene kodieren für wesentliche Komponenten der Zapfenphototransduktionskaskade:

1. die $\alpha$-Untereinheit des Zapfentransducins GNAT2 (ACHM4, OMIM \#613856) [12-15]: an der Guanosin-Bindungsstelle der Transducin- $\alpha$-Untereinheit wird GDP (Guanosindiphosphat) in GTP (Guansosintrisphosphat) umgewandelt,

2. die katalytische $\alpha$-Untereinheit der 3',5' cGMP-spezifischen Zapfenphosphodiesterase PDE6C (ACHM5, OMIM \#613093) $[16,17]$ : das Homodimer besteht aus $2 \alpha$-Untereinheiten und hydrolysiert cGMP. In Folge wird die cGMP-Konzentration im Außensegment der Zapfenphotorezeptorzelle gesenkt, 
3. die inhibitorische $\gamma$-Untereinheit der 3',5' cGMP-spezifischen Zapfenphosphodiesterase PDE6H (ACHM6, OMIM \#610024) [18],

4. die $\alpha$-(CNGA3 \{ACHM2, OMIM \#216900\})- $[19,20]$ und

5. die $\beta$-(CNGB3\{ACHM3, OMIM \#262300\})-Untereinheit des zapfenspezifischen cGMP-gesteuerten Kationenkanals (CNG, cyclic nucleotide-gated channel), der in der Plasmamembran des Zapfenaußensegments lokalisiert ist [21-23].

Mutationen in GNAT2, PDE6C und PDE6H kommen selten vor und wurden in weniger als $2 \%$ von Achromaten nachgewiesen [24]. Der Großteil, 70\% der humanen Achromatopsiefälle, wird durch „Channelopathien“, also Defekte in den Kanalproteinen CNGA3 oder CNGB3, verursacht [25-27]. Etwa 20-30\% der Erkrankten weisen Mutationen in der $\alpha$-Untereinheit des CNG-Ionenkanals der Zapfen auf, praktisch alle anderen liegen in der $\beta$-Untereinheit $[28,29]$. CNGA3 und CNGB3 kodieren jeweils für die $\alpha$ - und die $\beta$-Untereinheit des cGMP-gesteuerten Kationenkanals der Zapfen, der als Heterotetramer vorliegt [30,31]. Der CNG-Kanal nimmt eine Schlüsselposition im Sehprozess unter Tageslichtbedingungen ein, der mit der Lichtdetektierung in den Zapfenaußensegmenten beginnt [32]. Der CNG-Kanal kann als molekularer Schalter im Phototransduktionsprozess angesehen werden: lichtvermittelte Änderungen der cGMP-Konzentration (cGMP: zyklisches Guanosinmonophosphat) werden in ein Spannungssignal umgesetzt, das die Glutamatfreisetzung an der Zapfensynapse steuert [33]. Das neuronale Signal wird zunächst über Interneuronen (Bipolarzellen) an die Output-Neuronen (Ganglienzellen) der Netzhaut weitergeleitet. Die Ganglienzellen übermitteln die visuellen Informationen schließlich in Form von Aktionspotenzialen an den visuellen Kortex [34].

\section{Genersatztherapie der Achromatopsie: Voraussetzungen}

\section{Vektorsysteme}

Bei einer Genersatztherapie wird ein Krankheitszustand dadurch behandelt, dass man einen durch defekte Gene hervorgerufenen Mangel durch die Kopien gesunder, funktionsfähiger Gene beseitigt. Die Herausforderung einer solchen Therapie besteht in der Notwendigkeit, die Zielzellen mit der neuen genetischen Information unter Verwendung eines effizienten, sicheren Vektorsystems mit therapeutischer Langzeitwirkung auszustatten. Ein Vektorsystem, basierend auf rekombinanten Adeno-assoziierten Viren, hat sich sowohl aufgrund seiner Effizienz als auch seiner langen therapeutischen Wirkung in verschiedenen Therapieansätzen für Netzhauterkrankungen bewährt: nicht pathogene rekombinante Viren werden so modifiziert, dass sie mit einem Genkonstrukt bestehend aus der DNA-Sequenz des Targetgens und regulatorischen Sequenzen beladen werden [35,36]. Über eine subretinale Injektion erreichen die Viruspartikel die Photorezeptorschicht, transfizieren die Zielzellen der Netzhaut und setzen die neue genetische Information frei [37,38]. Diese kann nun in die Transkriptions- und Translationsprozesse der Sinneszelle eingebunden werden - im Idealfall übt das neue Protein seine Funktion in der Zielzelle, also in der von der Mutation betroffenen Photorezeptorzelle, aus. Verschiedene genetische Modulationen der viralen Eigenschaften als auch des Vektors können die Spezifität und Effizienz einer Genersatztherapie erhöhen [39]. So kann sowohl die Zellspezifität der rekombinanten modifizierten Viren unter Verwendung verschiedener Serotypen und Kapsidproteine als auch die Expressionsstärke, also wie viel neues Pro- tein letztendlich in der Zelle produziert wird, über regulatorische Sequenzen (Promotoren) gesteuert werden [40-45]. Ein anschauliches Beispiel für den Aufbau eines rAAV-Vektorkonstrukts, welches in der CNGA3-Gentherapie am Forschungsinstitut für Augenheilkunde in Tübingen verwendet wurde, ist in - Abb. 2 A dargestellt. Ein zellspezifischer Zapfenpromotor steuert in Zapfenzellen die Bildung von CNGA3. Flankiert wird die Sequenz von regulatorischen Elementen und viralen ITRs (Inverted Terminal Repeats), die u.a. die Replikation und die Verpackungseigenschaften bestimmen. Die modifizierten Viren persistieren als extrachromosomale Elemente und scheinen nicht in das Wirtsgenom zu integrieren $[46,47]$. Dadurch wird eine hohe Sicherheit von rAAV-vermittelter Therapie gewährleistet. Die Zellspezifität des Viruspartikels zur Oberfläche der Zapfenphotorezeptoren und die Eigenschaften, die den Beginn der Genexpression regulieren, werden über die Kombination von Kapsidprotein 5 und Serotyp 2 gesteuert [48-50]. In den letzten Jahren konnte in einer Vielzahl von gentherapeutischen Ansätzen am Tiermodell die erfolgreiche Behandlung erblicher Netzhautdystrophien unter Verwendung von rAAV-Vektoren gezeigt werden $[51,52]$. Letztendlich führten die positiven Wirkungen von rAAVSystemen, wie Langzeiteffekt der Therapie, Effizienz und Sicherheit, zu einer Reihe erster humaner Anwendungen bei Patienten mit Retinadystrophien, wie z.B. der Leber'schen kongenitalen Amaurose, LCA [53-55].

\section{Vektorapplikation: subretinale Injektion}

Viele retinale Dystrophien werden durch defekte Proteine in Photorezeptorzellen oder im retinalen Pigmentepithel (RPE) verursacht, sodass die beste Strategie zur Etablierung einer Gentherapie am Auge die lokale Gabe der therapeutisch wirksamen Virussuspension über eine subretinale Injektion ist. Das injizierte Material kann somit im subretinalen Bereich direkt mit den betroffenen Zellen in Kontakt treten. Das Prinzip ist in $\square$ Abb. $2 \mathbf{B}$ grafisch dargestellt: zwischen Neuroretina (mit Photorezeptoren) und dem RPE wird ein kleines Flüssigkeitsvolumen injiziert, das zu einer kurzzeitigen Ablatio der Netzhaut führt. In der Literatur sind im Tiermodell verschiedene Wege der subretinalen Applikation beschrieben: 1) der transkorneale Weg durch die Linse und den Glaskörper [56-58] oder 2) der transsklerale Weg pars plana am Limbus oder an der Ora serrata $[59,60]$. Beide Formen der subretinalen Applikation führen zur Transfektion von Photorezeptoren und zu einer als erfolgreich beschriebenen Genersatztherapie. Für die Wiederherstellung einer Netzhautfunktion im Tiermodell scheint jedoch der transsklerale Weg pars plana geeigneter. Mögliche Einschränkungen des Therapieerfolgs, bedingt durch eine Verletzung der Optik/Linse, sind unter Verwendung dieser Applikationsform weitgehend auszuschließen (ein detailliertes Injektionsprotokoll mit Hinweisen zur praktischen Durchführung ist unter „Retinal Degeneration: Methods and Protocols“ im Buchkapitel VII unter „Optimized Technique for Subretinal Injections in Mice“ zu finden [61]).

\section{Qualitätskontrolle subretinaler Injektion durch konfokale Scanning Laser-Ophthalmoskopie (cSLO)} und optische Kohärenztomografie (OCT)

Bedingt durch die Größe eines Mausauges ist der Erfolg einer subretinalen Injektion nicht immer leicht nachzuvollziehen. Bei der steigenden Zahl von Therapiestudien liegt der Fokus aber praktisch ausnahmslos auf der Weiterentwicklung und Verbesserung zellspezifischer und effizienter Vektorsysteme, nur ganz selten finden sich Angaben über eine Qualitätskontrolle der In- 


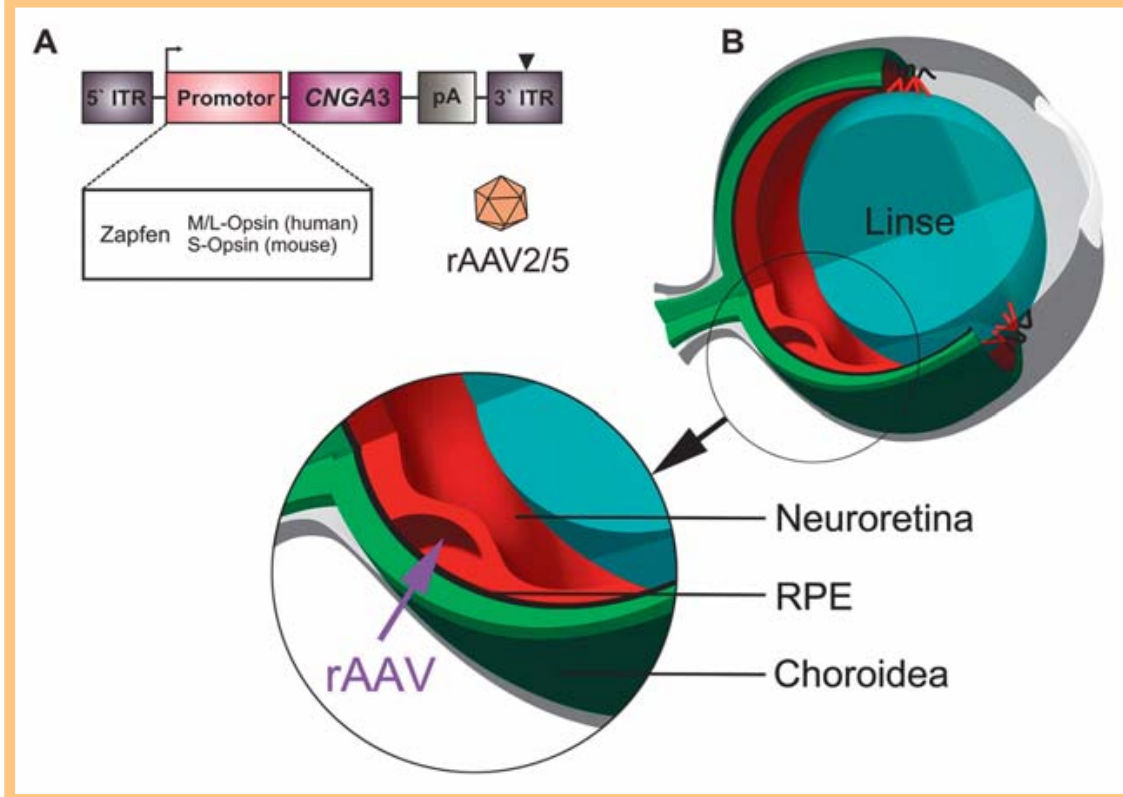

Abb. 2 A Schematische Darstellung der therapeutisch wirksamen Vektorsequenz, die in rAAVPartikel mit Serotyp 2 und Kapsidprotein 5 verpackt ist. CNGA3 wird unter einem zapfenspezifischen Promotor exprimiert. B Grafik eines subretinal injizierten Auges. Im Ausschnitt ist die Ablösung der Neuroretina (in rot) vom RPE (in schwarz) dargestellt, die kurzzeitig durch die Applikation der Virussuspension entsteht.

jektionen [62]. Daher bleibt in den meisten Studien offen, inwieweit die Qualität der Applikation das Ergebnis beeinflusst hat. Eine relativ gute primäre Kontrolle der intraokularen Prozedur gelingt direkt mit dem Operationsmikroskop. Für Langzeitstudien ist es jedoch wesentlich, nicht-invasive In-vivo-Diagnostikmethoden wie SLO und OCT [63-65] zu verwenden, um die Qualität der Injektion beurteilen und optimieren zu können. Die Morphologie der Netzhautschichten und des RPEs können direkt nach dem Eingriff und später nach definierten Zeitintervallen anhand von SLO-Fundusbildern und OCT-Scans evaluiert werden und liefern somit u.a. wertvolle Rückschlüsse auf die Qualität und Optimierungsoptionen der Behandlungstechnik.

\section{Therapieerfolgskontrolle durch Elektroretinografie (ERG)}

Das ERG ist eine etablierte diagnostische Technik der klinischen Ophthalmologie und beschreibt die elektrische Antwort der Retina auf Lichtreize. Die über die Elektroden abgeleiteten Potenziale der retinalen Zellen nach Stimulation mit standardisierten Lichtreizen verschiedener Intensität werden zunächst ohne (Bedingungen für skotopisches Sehen) und später mit Hintergrundbeleuchtung (Bedingungen für photopisches Sehen) gemessen und ausgewertet [66]. Anhand der ERG-Antworten ist es möglich, unmittelbare Schlüsse auf die Funktion und Integrität der Signaltransduktionswege der Retina zu ziehen. Die Technik findet nicht nur Einsatz in der Klinik, auch transgene Tiere, die das humane Krankheitsbild der Netzhautdystrophie widerspiegeln, werden auf ihre pathologisch veränderte Funktion hin untersucht [6769]. Elektrophysiologische Ableitungen der Retina werden ebenfalls von behandelten Tieren genommen, um die Funktionalität des therapeutisch wirksamen Proteins nachzuweisen. Als standardisiertes diagnostisches Verfahren sind die ERG-Daten therapierter Tiere zur Beurteilung des Therapieerfolgs von fundamentaler Bedeutung. Das ERG wurde in allen 4 Genersatztherapien für Achromatopsie, auf die im Folgenden ausführlicher eingegangen wird, als objektives Testsystem verwendet.

\section{Optomotorischer Test}

Der Vollständigkeit halber seien weitere Verfahren genannt, welche zur Kontrolle und Bewertung des Therapieerfolgs bei Achromatopsie am Modell angewandt werden. Messungen von Farbkontrastwahrnehmung und Sehschärfe erfolgen über den Vergleich der optomotorischen Reaktion behandelter versus nicht behandelter Tiere. Während des Tests befindet sich das Tier in einem zylindrischen Glasbehälter, um den herum ein 2., mit farbigen und grauen senkrechten Streifen versehener Glaszylinder konzentrisch rotiert. Die Farben Rot, Gelb, Grün und Blau, nach Intensität und Wellenlänge geeicht, werden jeweils in Kombination mit sämtlichen Helligkeitsstufen einer 16-stufigen Grauskala (intensitätsgeeicht und nach dem Prinzip der Konstanz der Unterschiedsschwellen zusammengestellt) durchgeprüft [70,71]. Somit kann unter photopischen Lichtbedingungen die spektrale Empfindlichkeit des Zapfensystems festgestellt und Rückschlüsse auf erfolgreich therapierte Zapfenzellen gezogen werden. Die hochgradige motorische Unruhe der Tiere bedingt häufig jedoch keine so klaren Resultate, da in der Regel Kopfbewegungen anstelle von Augenbewegungen ausgewertet werden müssen.

\section{Immunhistochemische Untersuchungen}

Morphologische wie auch immunhistochemische Ex-vivo-Analysen werden post mortem an präparierten Retinae durchgeführt und liefern wertvolle Hinweise über z.B. die Lokalisierung von Proteinen.

\section{Genersatztherapie von Achromatopsie: Ergebnisse im Tiermodell}

$\nabla$

\section{Gnat2 ${ }^{\text {cpfl3-Gentherapie }}$}

Alexander et al. beschrieben 2007 die erste rAAV-vermittelte gentherapeutische Studie an einem Tiermodell für Achroma-

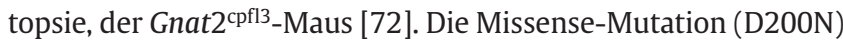
im $\alpha$-Transducin der Zapfenzelle führt zu einem Verlust der zapfenvermittelten Sehfunktion (keine photopischen ERG-Antworten), so wie es auch für Achromatopsie-Patienten bekannt ist [73]. Im gentherapeutischen Ansatz erhielten junge homozygote 


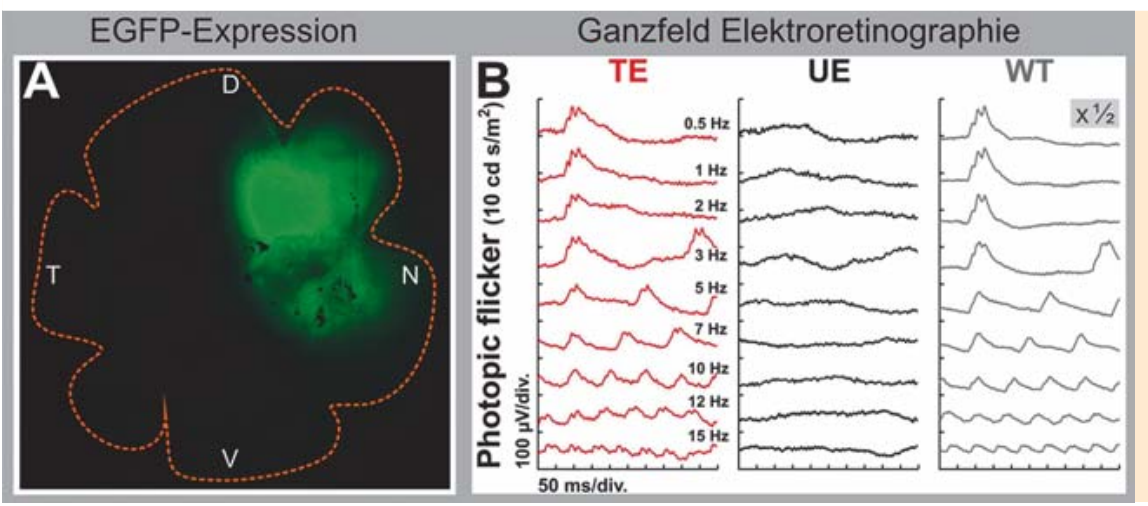

Abb.3 A Ganzpräparat einer Mausretina 8 Wochen nach subretinaler Applikation eines viral übertragenen Fluoreszenzfarbstoff-Gens EGFP (enhanced green fluorescent protein). Ca. $1 / 3-1 / 4$ der Retina wurde mittels rAAV-EGFP-Vektoren transfiziert. D: dorsal, V: ventral, T: temporal, N: nasal. B Elektrophysiologisches Korrelat der Sehverbesserung durch zapfenvermittelte Funktion in therapierten CNGA3-defizienten Mäusen unter Verwendung von rAAV-SWS-mCNGA3-Vektoren. Nicht behandeltes Auge (schwarz, UE), therapiertes Auge (rot, TE), Wildtypkontrolle (grau, WT). Ein deutlicher Funktionsgewinn der Zapfen nach Therapie konnte nachgewiesen werden.

Gnat ${ }^{\text {cpfl3 }}$-Tiere eine einmalige subretinale Injektion eines AAV5PR2.1-Gnat2-Vektors. Anwendung fand dabei ein humaner Rot/ Grün-Opsin-Promotor, der spezifisch die Expression vom murinen Gnat2-Gen in Zapfenzellen reguliert. Das funktionslose Protein wurde nun durch die Kopie eines gesunden Proteins ersetzt und führte 4 Wochen bis 7 Monate nach der Behandlung zu einem nachweislich zapfenvermittelten Funktionsgewinn („Rescue“). Die histomorphologischen Analysen der therapierten Retinae bestätigten die ERG-Daten.

\section{CNGB3-Gentherapie}

In ca. 50\% der untersuchten Achromatopsie-Patienten wurden Mutationen im CNGB3-Gen, in der $\beta$-Untereinheit des CNG-Kanals, identifiziert. Unter den mehr als 30 beschriebenen Mutationen weist die Mutation Thr383fsX mit über $80 \%$ die höchste Frequenz auf. Sie führt durch eine Leserasterverschiebung in der kodierenden DNA-Sequenz zum Verlust des carboxyterminalen Bereichs ab der Porenregion [74]. Der Fokus wissenschaftlicher Studien lag darin, eine Genersatztherapie am Tiermodell zu entwickeln, die für den Großteil der Achromatopsie-Patienten zu verwenden wäre. Mehrere Tiermodelle standen zur Entwicklung einer solchen Therapie zur Verfügung. Junge Cngb3-defiziente Mäuse erhielten eine einmalige subretinal applizierte Dosis von rAAV2/8-hCAR-hCNGB3-Vektoren. Die Sequenz des humanen CNGB3-Gens stand dabei unter der Regulation des humanen zapfenubiquitären Promotors Arrestin (hCAR). Vier Wochen bis 10 Monate nach Behandlung wurde der funktionelle Rescue des Zapfensystems im ERG nachgewiesen [75]. Um der Fragestellung nachzugehen, wie weit das therapeutische Zeitfenster in einer rAAV-vermittelten Therapie für CNGB3-Defizienz zu legen ist, wurden Tiere im Alter von 6, 15, 30, 90 Tagen und 6 Monaten injiziert. Einen Monat nach Behandlung wurde die Funktion des Zapfensystems mittels ERG untersucht. Es zeigte sich, dass zu jedem Alterszeitpunkt die Behandlung in zapfenspezifischen ERGSignalen resultierte. Die Daten weisen darauf hin, dass das therapeutische Zeitfenster trotz progressiver Degeneration der Zapfenzellen bis zu 6 Monaten relativ weit nach hinten gelegt werden kann. Zwei unabhängig voneinander auftretende, natürlich vorkommende, autosomal-rezessive CNGB3-Mutationen wurden in Hunden (Alaskan Malamute, Deutsch Kurzhaar) gefunden: $\mathrm{CNGB}^{\mathrm{m}} / \mathrm{m}$ mit einer Punktmutation in Exon 6 und CNGB3-/- mit einer Nullmutation bedingt durch die genomische Deletion des gesamten CNGB3-Gens [76-78]. Die Hunde zeigen einen der Achromatopsie ähnlichen Phänotypen, der sich zwischen 8-12 Wochen nach der Geburt entwickelt: Tagblindheit und das vorwiegende Fehlen retinaler Funktion unter photopischen Lichtbedingungen [79]. Im gentherapeutischen Ansatz wurden
rAAV5-Vektoren mit verschieden langen Sequenzen des humanen Rot/Grün-Opsin-Promotors (PR2.1 und 3LCR-PR0.5) verwendet, welche die Expression des humanen CNGB3 steuerten. Die Langzeitwirkung und Stabilität der Behandlung waren dabei unabhängig von der zugrunde liegenden Form der Mutation, jedoch spielten der gewählte Promotor und das Alter zu Beginn der Behandlung eine große Rolle im Therapieerfolg [80]. So führte die Anwendung der längeren Promotorsequenz in jüngeren Tieren (unter 28 Wochen) zu einem stabilen und lang anhaltenden Wiedergewinn der CNGB3-Proteinfunktion: ERG-Signale unter photopischen Bedingungen konnten bis zu 2,5 Jahre nach Behandlung gemessen werden, was auf lebenslang therapierte Zapfenzellen schließen lässt. Auch in diesem therapeutischen Ansatz wurde eine einmalige Vektordosis subretinal appliziert.

\section{CNGA3-Gentherapie (ACHM2)}

Michalakis et al. beschrieben 2010 in einer umfassenden Studie erstmalig eine rAAV-vermittelte Gentherapie der $\alpha$-Untereinheit des Kanalproteins CNGA3 [81]. In Form der CNGA3-knock-outMaus wurde mittels klassischer „gene knockout“-Technik das Exon 7 deletiert, das für die transmembranen Segmente 3-6 kodiert [82]. In Analogie zum Patienten zeigen die CNGA3-knockout-Mäuse einen zapfenspezifischen Funktionsverlust, der mit molekularen, strukturellen und morphologischen Veränderungen einhergeht, die letztlich zur Degeneration und zum Zelltod der betroffenen Zapfenzelle führen [83]. Somit stand ein geeignetes Achromatopsie-Tiermodell zur Verfügung, um eine Genersatztherapie mithilfe von rAAV zu etablieren. Zu Beginn des Projekts war nicht sicher, (1) ob es möglich wäre, den großen Membran-Protein-Komplex in den Außensegmenten der Zapfenzelle der Netzhaut exprimiert zu bekommen und (2) ob durch die Reexpression von CNGA3 die Zapfenzellen die fehlende Funktion wiedererlangen würden. In einer Kooperationsarbeit des Forschungsinstitutes für Augenheilkunde, Tübingen, des Departements Pharmazie der LMU München und dem Max-Planck-Institut für Neurobiologie in Martinsried, München konnte im Mausmodell nachgewiesen werden, dass das fehlende CNGA3-Gen erfolgreich in die funktionslosen Zapfen eingebracht werden konnte. Dazu wurden rAAV-Vektoren (rAAV2/5-SWS-mCNGA3) generiert, die eine Expression des murinen CNGA3 unter Kontrolle eines zapfenspezifischen S-Opsin-Promotors ermöglichten. Diese therapeutischen Viren wurden in junge CNGA3-knock-out-Mäuse einseitig subretinal injiziert. Nach 8 Wochen konnte eine rAAV-vermittelte Expression von CNGA3 nachgewiesen werden. Das exogene CNGA3-Protein war in der Lage, mit endogenen CNGB3-Untereinheiten Heteromere zu bilden, welche in das Außensegment transportiert wurden. Diese neu generierten Ka- 
nalkomplexe konnten die bis dato inaktive Phototransduktionskaskade in den funktionslosen Zapfen aktivieren, um erstmals die Generierung zapfenvermittelter Lichtantworten zu ermöglichen ( Abb. 3). Mittels ERG und Ableitung von Aktionspotenzialen konnte außerdem gezeigt werden, dass diese neuen zapfenvermittelten Lichtinformationen an nachgeschaltete Bipolar- und Ganglienzellen geleitet werden. Die Ganglienzellen waren nun in der Lage, zapfenspezifische lichtabhängige Aktionspotenziale zu generieren und an das Gehirn zu übermitteln. Diese Ergebnisse verdeutlichten, dass die Netzhaut nach erfolgreicher Genersatztherapie zapfenvermittelte lichtabhängige Signale an das Gehirn sendet. Da die Tiere zapfenblind geboren wurden, stellte sich die Frage, ob das Gehirn nun nach Therapie in der Lage ist, diese „neuen“ Signale zu verarbeiten? Zur Beantwortung dieser Frage wurden die therapierten Mäuse auf ihre Fähigkeit hin getestet, zwischen 2 Objekten mit unterschiedlicher spektraler Information zu unterscheiden. Dazu wurden die Mäuse trainiert, eine stabile Plattform, die durch einen roten Karton gekennzeichnet war, in einem Wasserbecken zu finden und diese von einer 2. Plattform, die mit einem grünen Karton markiert war und untergeht, sobald die Maus auf diese klettert, zu unterscheiden. Die therapierten Mäuse waren, ähnlich wie die Kontrollmäuse, in der Lage, zwischen beiden Farben zu unterscheiden und die richtige (stabile) Plattform zu finden. Hingegen zeigten nicht therapierte CNGA3-knock-out-Mäuse keine Präferenz für die richtige Plattform. Der in vivo nachgewiesene Therapieerfolg zeigte sich in der Wiederherstellung der komplett fehlenden Funktion des Zapfensystems auf der Ebene der Retina bis hin zur kortikalen Wahrnehmung von Farbkontrasten. Gleichzeitig bewirkte die Therapie eine Reduktion der Degeneration entsprechender Netzhautzellen. Mit dieser umfangreichen tierexperimentellen Studie wurde erstmalig ein Proof-of-Principle einer Genersatztherapie für Achromatopsie erbracht: das war der Startpunkt des ersten Translationsprojekts bei einer erblichen Netzhauterkrankung in Deutschland (http://www.rd-cure.de).

\section{cpfl5-Gentherapie}

Ein okularer Phänotyp ähnlich dem der Achromatopsie wurde für die cpfl5-Maus (cone photoreceptor function loss 5), einer natürlichen Mutante mit einer Punktmutation im Exon 5 des CNGA3-Gens beschrieben [84]. Auch für dieses Tiermodell wurde eine rAAV-vermittelte Gentherapie entwickelt. Als Vektor wurde ein humaner Blauzapfen-Opsin-Promotor HB570 verwendet, der die Expression des murinen CNGA3-Gens reguliert (AAV5-CBAmCNGA3). Eine einmalige subretinale Injektion wurde in jungen cpfl5-Mäusen durchgeführt und der Therapieeffekt nach 3 Wochen, 3 und 5 Monaten im ERG und im optomotorischen Test untersucht. Unter photopischen Lichtbedingungen zeigte sich in den behandelten Tieren 5 Monate nach Behandlung ein bis zu $60 \%$ starker Funktionsgewinn in zapfenspezifischen ERG-Antworten im Vergleich zu den Wildtypen. Das CNGA3-Protein mit einer korrekten Lokalisierung im Außensegment wurde mittels immunohistochemischer Analysen im behandelten Bereich nachgewiesen [85].

\section{Diskussion und Ausblick}

\section{$\nabla$}

Durch seine freie Zugänglichkeit, seine Kompartimentierung und seinen Status als immunprivilegierte Zone kann das Auge als ein hervorragendes Modellsystem für die Entwicklung einer Gentherapie erblicher Netzhauterkrankungen genutzt werden. Die größte Herausforderung und anstehende Aufgabe im Bereich der Gentherapie der Achromatopsie ist die Translation bisheriger Ergebnisse in den Bereich der Humanmedizin und die Initiierung entsprechender klinischer Studien. Eine neue Ära retinaler Therapieansätze ist durch die bisherigen Erfolge der RPE65-Defizienz in tierexperimentellen gentherapeutischen Studien eingeleitet worden und führte zur humanen Translation. Momentan laufen weltweit 4 voneinander unabhängige klinische Studien zur Behandlung einer frühen und schweren Form der hereditären Netzhautdystrophien (LCA), der ein Vitamin-A-Mangel zugrunde liegt (Phasen I \& II, http://clinicaltrials.gov/ct2/results?term=eye+gene +therapy+RPE65). Eine Genersatztherapie mittels rAAV-Vektoren (Virus-Serotype 2, rAAV2) führte in 7 von 9 Patienten, die einen angeborenen Defekt im RPE65-Gen tragen, zu einer Verbesserung der visuellen Funktion. Langzeitstudien über einen Zeitraum von 5 Jahren zeigen erfolgversprechende Ergebnisse über einen stabilen Funktionsgewinn und ermöglichten eine Beurteilung der Sicherheit der viralen Therapie [86-88]. Basierend auf diesen Ergebnissen wurden ebenfalls in tierexperimentellen Studien Genersatztherapien für Achromatopsie entwickelt. Verschiedene technische Methoden zur Erhöhung und Spezifität der rAAV-Transduktion wurden in vivo untersucht: 1) Verwendung zellspezifischer Promotoren, 2) Entwicklung von rAAV-Serotypen und Kapsidproteinen sowie 3) Zugabe therapeutisch wirksamer Medikamente [89]. Es zeigte sich in allen 4 beschriebenen Genersatztherapien am Tiermodell, dass die verwendeten Kombinationen aus Promotorsequenz, Serotyp und Kapsidprotein zur Expression des Ersatzgens führte. Mehrere Optimierungsstrategien wurden evaluiert. Komáromy et al. verwendeten für die Therapie CNGB3-defizienter Hunde 2 verschieden lange Sequenzen des Rot/Grün-Opsin-Promotors und stellten Unterschiede in der Langzeitwirkung und Stabilität der Behandlung fest. So führte die Anwendung der kürzeren Promotorsequenz zu einer transienten und sehr schwachen Expression des CNGB3-Gens. Der Einsatz der längeren Promotorsequenz in jüngeren Tieren hingegen hatte einen stabilen und lang anhaltenden Wiedergewinn der CNGB3-Proteinfunktion zur Folge. Abhängig von der Pathogenese des Tiermodells und den Varianten aus regulatorischen Einheiten und viralen Eigenschaften lassen sich demzufolge Strategien entwickeln, die zu einer optimierten Genersatztherapie führen. In der Tübinger-Münchner Studie führte eine einmalige subretinale Injektion eines kleinen Flüssigkeitsvolumens viraler Partikel zu einer lebenslang wirksamen Therapie. Nicht nur die Sehfunktion der Zapfen wurde wiederhergestellt, auch der Degenerationsprozess retinaler Zellen wurde verzögert. Der funktionelle Wiedergewinn des Proteins weist auf 1) einen korrekten Translationsprozess, 2) den Transport in die Zapfenaußensegmente und 3) die Integration in und Aktivierung der Phototransduktionskaskade hin. In den verschiedenen Studien wurde, im Vergleich zu den Kontrolltieren, eine unterschiedliche Stärke des Funktionsgewinns, die jedoch immer unter $100 \%$ lag, beschrieben. Die Ursachen hierfür könnten sehr mannigfaltig sein. Extrapolierte Daten aus unseren Studien zeigen, dass die Stärke des funktionellen Rescues mit der Größe des behandelten Bereichs durchaus in Beziehung gesetzt werden kann (siehe $\bullet$ Abb. 3, unpublizierte Ergebnisse). Ein funktioneller Rescue von ca. 30\%, der im Mittel erreicht wurde, war durchaus ausreichend, um zapfenspezifische Lichtsignale zu generieren, diese in Aktionspotenziale umzuwandeln und als visuelle Information im Kortex zu einem zapfengesteuerten Verhalten zu verarbeiten. Fraglich wäre nun, ob eine $100 \%$ ige Transfektion aller retinalen Zellen anzustreben wäre, da sie entweder nur durch 
mehrmalige Injektionen oder ein größeres Flüssigkeitsvolumen zu erreichen ist. Beide Möglichkeiten könnten jedoch in Folge irreparable Schäden am Auge nach sich ziehen. Des Weiteren wurden im Großteil der Studien zur Therapie von Achromatopsie Vektoren verwendet, die humane DNA-Sequenzen enthielten und in murinen bzw. caninen Tiermodellen um Einsatz kamen. Die Anwendung xenogener, also artfremder DNA-Sequenzen des Promotors und des Ersatzgens könnten die Regulierung der Transkription und Expression von Genen beeinflussen. Die Proteine der retinalen Phototransduktionskaskade sind jedoch zwischen den Spezies so hoch konserviert, dass von keinem bedeutenden Sequenzunterschied auszugehen ist, der die Expression hätte beeinflussen können. Die Wahl des Alters und die damit verbundene Progression der Degeneration zum Zeitpunkt der Behandlung sind sicherlich von größerer Bedeutung für den Erfolg einer Gentherapie anzusehen. Es konnte gezeigt werden, dass Defekte des CNGA3- wie auch des CNGB3-Kanals in jungen Tieren (ab postnatal 12 Tage) wie auch in älteren Tieren (bis zu 6 Monate) therapierbar sind. Das lässt die Schlussfolgerung zu, dass auch erwachsene Achromatopsie-Patienten mit progressivem Verlust an Zapfenzellen im therapeutischen Zeitfenster liegen können. Die hier aufgeführten Methoden der Vektorapplikation und nicht invasiven Diagnostik zur Qualitäts- und Erfolgskontrolle sind allesamt für den Einsatz am Menschen geeignet und können bei der humanen Translation Einsatz finden. Subretinale Injektionen am Menschen werden täglich in der Klinik durchgeführt, und die bisherigen Ergebnisse der humanen RPE65-Studien weisen auf eine hohe Sicherheit, wenig immunologische Reaktion und keine Nebenwirkungen unter Anwendung von rAAV-Vektoren hin. Elektrophysiologische wie auch morphologische Untersuchungen (ERG, SLO, OCT) werden routinemäßig in der ophthalmologischen Diagnostik eingesetzt. Aufgrund ihres nicht-invasiven Charakters führen sie zu keinen gesundheitlichen Beeinträchtigungen. Besonders die Rolle der optischen Kohärenztomografie bei der Qualitätskontrolle des operativen Eingriffs sei hier hervorgehoben.

Die Fortschritte, Erfolge und technischen Weiterentwicklungen von viralen Vektoren führten nun zum Start der ersten gentherapeutischen Studie zur Behandlung von Achromatopsie-Patienten in Deutschland. Auf der Basis umfangreich gesicherter tierexperimenteller Daten konnte ein Proof-of-Principle erarbeitet werden: von Geburt an zapfenblinde Mäuse erwarben eine komplett fehlende Sinnesqualität, das zapfenvermittelte Sehen. Therapierte Photorezeptoren generierten reguläre Signale, die in einem von Zapfensehen beeinflussten Verhalten resultierten und lebenslang wirkten. Im humanen Translationsprozess werden nun sowohl der Einsatz und die Auswahl humaner Vektoren als auch die Sicherheit und Effizienz des ausgewählten Vektorsystems evaluiert, um einen optimalen Therapieansatz für den Patienten bereitzustellen.

\section{Danksagung}

$\nabla$

Wir danken der Deutschen Forschungsgemeinschaft (Fördernummern: Se837/5-2, Se837/6-2, Se837/7-1) und der Tistou \& Charlotte Kerstan Stiftung (RD-CURE) für die finanzielle Unterstützung.

\section{Interessenkonflikt}

$\nabla$

Nein.

\section{Literatur}

1 Sohocki MM, Daiger SP, Bowne SJ et al. Prevalence of mutations causing retinitis pigmentosa and other inherited retinopathies. Hum Mutat 2001; 17: 42-51

2 Rattner A, Sun H, Nathans J. Molecular genetics of human retinal disease. Annu Rev Genet 1999; 33: 89-131

3 Berger W, Kloeckener-Gruissem B, Neidhardt J. The molecular basis of human retinal and vitreoretinal diseases. Prog Retin Eye Res 2010; 29: 335-375

4 Surace EM, Auricchio A. Versatility of AAV vectors for retinal gene transfer. Vision Res 2008; 48: 353-359

5 Thiadens AA, Phan TM, Zekveld-Vroon RC et al. Clinical course, genetic etiology, and visual outcome in cone and cone-rod dystrophy. Ophthalmology 2012; 119: 819-826

6 Francois J. Heredity in Ophthalmology. CV Mosby: St Louis; 1961

7 Sharpe LT, Nordby K. Total Colour Blindness: An Introduction. In: Hess RF, Sharpe LT, Nordby K, eds. Night Vision: basic, clinical and applied Aspects. Cambridge, UK: Cambridge University Press; 1990: 253-289

8 Sharpe LT, Stockman A, Jägle H et al. Opsin Genes, Cone Photopigments and Colour Blindness. In: Gegenfurtner KR, Sharpe LT, eds. Color Vision: From Genes to Perception. Cambridge, UK: Cambridge University Press; 1999

9 Park WL, Sunness JS. Red contact lenses for alleviation of photophobia in patients with cone disorders. Am J Ophthalmol 2004; 137: 774-775

10 Schornack $M$. Prescription and management of contact lenses in patients with monocular visual impairment. Optometry 2007; 78: 652656

11 Young RS, Krefman RA, Fishman GA. Visual improvements with redtinted glasses in a patient with cone dystrophy. Arch Ophthalmol 1982; 100: 268-271

12 Kohl S, Baumann B, Rosenberg T et al. Mutations in the cone photoreceptor G-protein alpha-subunit gene GNAT2 in patients with achromatopsia. Am J Hum Genet 2002; 71: 422-425

13 Aligianis IA, Forshew T, Johnson $S$ et al. Mapping of a novel locus for achromatopsia (ACHM4) to $1 \mathrm{p}$ and identification of a germline mutation in the alpha subunit of cone transducin (GNAT2). J Med Genet 2002; 39: 656-660

14 Rosenberg T, Baumann B, Kohl S et al. Variant phenotypes of incomplete achromatopsia in two cousins with GNAT2 gene mutations. Invest Ophthalmol Vis Sci 2004; 45: 4256-4262

15 Ouechtati F, Merdassi A, Bouyacoub Y et al. Clinical and genetic investigation of a large Tunisian family with complete achromatopsia: identification of a new nonsense mutation in GNAT2 gene. J Hum Genet 2011; 56: 22-28

16 Chang B, Grau T, Dangel S et al. A homologous genetic basis of the murine cpfl1 mutant and human achromatopsia linked to mutations in the PDE6C gene. Proc Natl Acad Sci U S A 2009; 106: 19581-19586

17 Thiadens AA, den Hollander AI, Roosing $S$ et al. Homozygosity mapping reveals PDE6C mutations in patients with early-onset cone photoreceptor disorders. Am J Hum Genet 2009; 85: 240-247

18 Kohl S, Coppieters F, Meire F et al. A nonsense mutation in PDE6H causes autosomal-recessive incomplete achromatopsia. Am J Hum Genet 2012; 91: 527-532

19 Wissinger B, Gamer D, Jägle $H$ et al. CNGA3 mutations in hereditary cone photoreceptor disorders. Am J Hum Genet 2001; 69: 722-737

$20 \mathrm{Kohl}$ S, Marx T, Giddings I et al. Total colourblindness is caused by mutations in the gene encoding the alpha-subunit of the cone photoreceptor cGMP-gated cation channel. Nat Genet 1998; 19: 257-259

21 Sundin OH, Yang JM, Li Y et al. Genetic basis of total colourblindness among the Pingelapese islanders. Nat Genet 2000; 25: 289-293

22 Winick JD, Blundell ML, Galke BL et al. Homozygosity mapping of the Achromatopsia locus in the Pingelapese. Am J Hum Genet 1999; 64: 1679-1685

23 Milunsky A, Huang XL, Milunsky J et al. A locus for autosomal recessive achromatopsia on human chromosome 8q. Clin Genet 1999; 56: 82-85

24 Grau T, Artemyev NO, Rosenberg T et al. Decreased catalytic activity and altered activation properties of PDE6C mutants associated with autosomal recessive achromatopsia. Hum Mol Genet 2011; 20: 719-730 
25 Kohl S, Varsanyi B, Antunes GA et al. CNGB3 mutations account for 50\% of all cases with autosomal recessive achromatopsia. Eur J Hum Genet 2005; 13: 302-308

26 Michaelides M, Aligianis IA, Ainsworth JR et al. Progressive cone dystrophy associated with mutation in CNGB3. Invest Ophthalmol Vis Sci 2004; 45: 1975-1982

27 Thiadens AA, Slingerland NW, Roosing S et al. Genetic etiology and clinical consequences of complete and incomplete achromatopsia. Ophthalmology 2009; 116: 1984-1989

28 Kohl S, Kitiratschky V, Papke M et al. Genes and mutations in autosomal dominant cone and cone-rod dystrophy. Adv Exp Med Biol 2012; 723: 337-343

29 Johnson S, Michaelides M, Aligianis IA et al. Achromatopsia caused by novel mutations in both CNGA3 and CNGB3. J Med Genet 2004; 41: e20

30 Kaupp UB, Seifert R. Cyclic nucleotide-gated ion channels. Physiol Rev 2002; 82: 769-824

31 Zhong H, Molday LL, Molday RS et al. The heteromeric cyclic nucleotidegated channel adopts a 3A:1B stoichiometry. Nature 2002; 420: 193198

32 Zagotta WN, Siegelbaum SA. Structure and function of cyclic nucleotide-gated channels. Annu Rev Neurosci 1996; 19: 235-263

33 Hofmann F, Biel M, Kaupp UB et al. International Union of Pharmacology. XLII. Compendium of voltage-gated ion channels: cyclic nucleotide-modulated channels. Pharmacol Rev 2003; 55: 587-589

34 Hofmann F, Biel M, Kaupp UB. International Union of Pharmacology. LI. Nomenclature and structure-function relationships of cyclic nucleotide-regulated channels. Pharmacol Rev 2005; 57: 455-462

35 Ali RR, Reichel MB, Thrasher AJ et al. Gene transfer into the mouse retina mediated by an adeno-associated viral vector. Hum Mol Genet 1996; 5: 591-594

36 Allocca M, Mussolino C, Garcia-Hoyos M et al. Novel adeno-associated virus serotypes efficiently transduce murine photoreceptors. J Virol 2007; 81: 11372-11380

37 Bainbridge JW, Mistry A, Schlichtenbrede FC et al. Stable rAAV-mediated transduction of rod and cone photoreceptors in the canine retina. Gene Ther 2003; 10: 1336-1344

38 Buch PK, Bainbridge JW, Ali RR. AAV-mediated gene therapy for retinal disorders: from mouse to man. Gene Ther 2008; 15: 849-857

39 Pang JJ, Lauramore A, Deng WT et al. Comparative analysis of in vivo and in vitro AAV vector transduction in the neonatal mouse retina: effects of serotype and site of administration. Vision Res 2008; 48: 377-385

40 Chen J, Tucker CL, Woodford B et al. The human blue opsin promoter directs transgene expression in short-wave cones and bipolar cells in the mouse retina. Proc Natl Acad Sci U S A 1994; 91: 2611-2615

41 Yang GS, Schmidt M, Yan Z et al. Virus-mediated transduction of murine retina with adeno-associated virus: effects of viral capsid and genome size. J Virol 2002; 76: 7651-7660

42 Glushakova LG, Timmers AM, Pang J et al. Human blue-opsin promoter preferentially targets reporter gene expression to rat s-cone photoreceptors. Invest Ophthalmol Vis Sci 2006; 47: 3505-3513

43 Komáromy AM, Alexander JJ, Cooper AE et al. Targeting gene expression to cones with human cone opsin promoters in recombinant AAV. Gene Ther 2008; 15: 1049-1055; Erratum in: Gene Ther 2008; 15:1073; Glushakova LG [added]. Gene Ther 2011; 18: 1179

44 Lebherz C, Maguire A, Tang $W$ et al. Novel AAV serotypes for improved ocular gene transfer. J Gene Med 2008; 10: 375-382

45 Smith $R H$. Adeno-associated virus integration: virus versus vector. Gene Therapy 2008; 15: 817-822

46 Schnepp BC, Jensen RL, Chen CL et al. Characterization of adeno-associated virus genomes isolated from human tissues. J Virol 2005; 79: 14793-14803

47 Grieger JC, Samulski RJ. Adeno-associated virus vectorology, manufacturing, and clinical applications. Methods Enzymol 2012; 507: 229254

48 Surace EM, Auricchio A. Versatility of AAV vectors for retinal gene transfer. Vision Res 2008; 48: 353-359

49 Petrs-Silva H, Dinculescu A, Li Q et al. High-efficiency transduction of the mouse retina by tyrosine-mutant AAV serotype vectors. Mol Ther 2009; 17: 463-471

50 Allocca $M$, Doria $M$, Petrillo $M$ et al. Serotype-dependent packaging of large genes in adeno-associated viral vectors results in effective gene delivery in mice. J Clin Invest 2008; 118: 1955-1964

51 Acland GM, Aguirre GD, Ray J et al. Gene therapy restores vision in a canine model of childhood blindness. Nat Genet 2001; 28: 92-95
52 Ali RR, Sarra GM, Stephens C et al. Restoration of photoreceptor ultrastructure and function in retinal degeneration slow mice by gene therapy. Nat Genet 2000; 25: 306-310

53 Bainbridge JW, Smith AJ, Barker SS et al. Effect of gene therapy on visual function in Leber's congenital amaurosis. N Engl J Med 2008; 358: 2231-2239

54 Cideciyan AV, Aleman TS, Boye SL et al. Human gene therapy for RPE65 isomerase deficiency activates the retinoid cycle of vision but with slow rod kinetics. Proc Natl Acad Sci U S A 2008; 105: 15112-15117

55 Hauswirth WW, Aleman TS, Kaushal S et al. Treatment of leber congenital amaurosis due to RPE65 mutations by ocular subretinal injection of adeno-associated virus gene vector: short-term results of a phase I trial. Hum Gene Ther 2008; 19: 979-990

56 Timmers AM, Zhang H, Squitieri A et al. Subretinal injections in rodent eyes: effects on electrophysiology and histology of rat retina. Mol Vis 2001; 7: 131-137

57 Johnson CJ, Berglin L, Chrenek MA et al. Technical brief: subretinal injection and electroporation into adult mouse eyes. Mol Vis 2008; 14: 2211-2226

58 Busskamp V, Duebel J, Balya D et al. Genetic reactivation of cone photoreceptors restores visual responses in retinitis pigmentosa. Science 2010; 329: 413-417

59 Price J, Turner D, Cepko C. Lineage analysis in the vertebrate nervous system by retrovirus-mediated gene transfer. Proc Natl Acad Sci U S A 1987; 84: 156-160

60 Schlichtenbrede FC, da Cruz L, Stephens C et al. Long-term evaluation of retinal function in Prph2Rd2/Rd2 mice following AAV-mediated gene replacement therapy. J Gene Med 2003; 5: 757-764

61 Mühlfriedel R, Michalakis S, Garrido MG et al. Optimized technique for subretinal injections in mice. Methods Mol Biol 2013; 935: 343-349

62 Liang FQ Anand V, Maguire AM et al. Intraocular delivery of recombinant virus. Methods Mol Med 2001; 47: 125-139

63 Seeliger MW, Beck SC, Pereyra-Muñoz N et al. In vivo confocal imaging of the retina in animal models using scanning laser ophthalmoscopy. Vision Res 2005; 45: 3512-3519

64 Huber G, Beck SC, Grimm C et al. Spectral domain optical coherence tomography in mouse models of retinal degeneration. Invest Ophthalmol Vis Sci 2009; 50: 5888-5895

65 Fischer MD, Huber G, Beck SC et al. Noninvasive, in vivo assessment of mouse retinal structure using optical coherence tomography. PLoS One 2009; 4: e7507

66 Marmor MF, Fulton AB, Holder GE et al; International Society for Clinical Electrophysiology of Vision. MISCEV Standard for full-field clinical electroretinography (2008 update). Doc Ophthalmol 2009; 118: 69-77

67 Scholl HP, Zrenner E. Electrophysiology in the investigation of acquired retinal disorders. Surv Ophthalmol 2000; 45: 29-47

68 Dalke C, Löster J, Fuchs H et al. Electroretinography as a screening method for mutations causing retinal dysfunction in mice. Invest Ophthalmol Vis Sci 2004; 45: 601-609

69 Tanimoto N, Muehlfriedel RL, Fischer MD et al. Vision tests in the mouse: Functional phenotyping with electroretinography. Front Biosci (Landmark Ed) 2009; 14: 2730-2737

70 Douglas RM, Alam NM, Silver BD et al. Independent visual threshold measurements in the two eyes of freely moving rats and mice using a virtual-reality optokinetic system. Vis Neurosci 2005; 22: 677-684

71 Umino $Y$, Solessio E, Barlow RB. Speed, spatial, and temporal tuning of rod and cone vision in mouse. J Neurosci 2008; 28: 189-198

72 Alexander JJ, Umino Y, Everhart D et al. Restoration of cone vision in a mouse model of achromatopsia. Nat Med 2007; 13: 685-687

73 Deeb SS. Molecular genetics of color-vision deficiencies. Vis Neurosci 2004; 21: 191-196

74 Wiszniewski W, Lewis RA, Lupski JR. Achromatopsia: the CNGB3 p. T383fsX mutation results from a founder effect and is responsible for the visual phenotype in the original report of uniparental disomy 14 . Hum Genet 2007; 121: 433-439

75 Carvalho LS, Xu J, Pearson RA et al. Long-term and age-dependent restoration of visual function in a mouse model of CNGB3-associated achromatopsia following gene therapy. Hum Mol Genet 2011; 20: 3161-3175

76 Aguirre GD, Rubin LF. Pathology of hemeralopia in the Alaskan malamute dog. Invest Ophthalmol 1974; 13: 231-235

77 Aguirre GD, Rubin LF. The electroretinogram in dogs with inherited cone degeneration. Invest Ophthalmol 1975; 14: 840-847

78 Rubin LF. Clinical features of hemeralopia in the adult Alaskan malamute. J Am Vet Med Assoc 1971; 158: 1696-1698 
79 Sidjanin DJ, Lowe JK, McElwee JL et al. Canine CNGB3 mutations establish cone degeneration as orthologous to the human achromatopsia locus ACHM3. Hum Mol Genet 2002; 11: 1823-1833

80 Komáromy AM, Alexander JJ, Rowlan JS et al. Gene therapy rescues cone function in congenital achromatopsia. Hum Mol Genet 2010; 19: 2581-2593; Erratum in: Hum Mol Genet 2011; 20: 5024

81 Michalakis S, Mühlfriedel R, Tanimoto $N$ et al. Restoration of cone vision in the CNGA3-/- mouse model of congenital complete lack of cone photoreceptor function. Mol Ther 2010; 18: 2057-2063

82 Biel M, Seeliger M, Pfeifer A et al. Selective loss of cone function in mice lacking the cyclic nucleotide-gated channel CNG3. Proc Natl Acad Sci U S A 1999; 96: 7553-7557

83 Michalakis S, Geiger H, Haverkamp S et al. Impaired opsin targeting and cone photoreceptor migration in the retina of mice lacking the cyclic nucleotide-gated channel CNGA3. Invest Ophthalmol Vis Sci 2005; 46: 1516-1524

84 Hawes NL, Wang X, Hurd RE. A point mutation in the Cnga3 gene causes cone photoreceptor function loss (Cpfl5) in mice. Paper presented at ARVO, E-Abstract 4579: 2006, Fort Lauderdale; 2006
85 Pang JJ, Deng WT, Dai X et al. AAV-mediated cone rescue in a naturally occurring mouse model of CNGA3-achromatopsia. PLoS One 2012; 7: e35250

86 Maguire AM, Simonelli F, Pierce EA et al. Safety and efficacy of gene transfer for Leber's congenital amaurosis. N Engl J Med 2008; 358: 2240-2248

87 Amado D, Mingozzi F, Hui D et al. Safety and efficacy of subretinal readministration of a viral vector in large animals to treat congenital blindness. Sci Transl Med 2010; 2: 21ra16

88 Jacobson SG, Cideciyan AV, Ratnakaram $R$ et al. Gene therapy for leber congenital amaurosis caused by RPE65 mutations: safety and efficacy in 15 children and adults followed up to 3 years. Arch Ophthalmol 2012; 130: 9-24

89 Zhang S, Wu J, Wu X et al. Enhancement of rAAV2-mediated transgene expression in retina cells in vitro and in vivo by coadministration of low-dose chemotherapeutic drugs. Invest Ophthalmol Vis Sci 2012; 53: $2675-2684$ 